\title{
Pediatric E.N.T. emergencies during COVID-19 pandemic: our experience
}

\author{
Anand Palas $^{1} \cdot$ Jayman Raval $^{1}\left(\mathbb{D} \cdot\right.$ R. G. Aiyer ${ }^{1} \cdot$ Bhagyalakshmi Arunlal $^{1}$
}

Received: 5 October 2020/Accepted: 23 December 2020/Published online: 4 January 2021

(C) Association of Otolaryngologists of India 2021

\begin{abstract}
Our aim is to analyze effect of covid-19 pandemic in management of pediatric emergencies in E.N.T. and to provide recommendations for management of pediatric emergencies in E.N.T. during pandemic. In this retrospective study we included emergency pediatric cases required early intervention during covid-19 pandemic. Pre operative radiological investigation $\mathrm{x}$-ray was done in all aero digestive foreign body patients. Pre operative HRCT neck and chest with virtual bronchoscopy in foreign body inhalation \& compromised airway patients was done only in cases where patient's clinical condition was stable. Similarly RT PCR for covid-19 screening done only in cases where patient's clinical condition was stable. Adequate personal protective equipment was used during all the procedures. Total 29 Pediatric patients age ranging from 1 day to 13 years underwent procedures due to history of foreign body inhalation; ingestion, insertion and compromised airway in form of immediate endotracheal intubation were included. Comprehensively we recommend intervention only in emergency procedures during pandemic, use of personal protective equipment during all procedures, preoperative investigations for diagnosis and modifications in operation theatre, anesthesia and surgical techniques to reduce aerosols generation will minimize risk of infection transmission to health care workers.
\end{abstract}

Keywords COVID-19 · Pediatric emergencies . Aerosol generating procedure $\cdot$ Pediatric airway

Jayman Raval

dr.jaymanraval11@gmail.com

1 Department of E.N.T. and Head-Neck Surgery, Baroda Medical College and S.S.G. Hospital, Vadodara, Gujarat, India

\section{Introduction}

The worldwide first case of corona virus disease 2019 (COVID-19) was identified in December 2019 in Wuhan, Hubei province, china. COVID-19 has low fatality rate as compared to severe acute respiratory syndrome (SARS) and Middle East respiratory syndrome (MERS) but higher transmission rate, long incubation period and asymptomatic spread of severe acute respiratory syndrome corona virus-2 (SARS-CoV-2) have resulted in a large number of deaths [1]. The World Health Organization (WHO) had announced COVID-19 outbreak a pandemic since March 11, 2020

COVID-19 spreads primarily through the respiratory tract by virus particles in droplets and aerosols [2]. Since procedures and examinations involving upper aero digestive tract have a high probability to generate aerosols, E.N.T. surgeons are at particularly higher risk for transmission [3]. Various professional bodies have recommended that only emergency procedures should be performed and appropriate personal protective equipment (PPE) is necessary during the COVID-19 pandemic [4].

Aero digestive tract and ear foreign bodies in pediatric age group are most common emergencies in E.N.T [5]." Also pediatric patients of compromised airway frequently referred to E.N.T. surgeon. Such emergencies may cause fatal outcome, if not intervened timely. To deal with such airway emergencies in pediatric patients during covid-19 pandemic is in itself a challenging task.

In this study we are sharing our institutional experience for management of pediatric E.N.T. emergencies during covid-19 pandemic and to provide recommendations for management of pediatric emergencies in E.N.T. during pandemic. 


\section{Methods}

The present retrospective study was carried out in Department of E.N.T. and Head-Neck surgery, Medical College Baroda during covid-19 pandemic from 22/03/ 2020 to 30/06/2020. Emergency pediatric cases require early intervention during covid-19 pandemic were included. All procedures performed in the studies involving human participant were in accordance with the ethical standards of the institutional research committee and ethical standards.

All these patients were presented to E.N.T. or pediatric OPD and emergency department. Detailed clinical history and clinical examination was carried out using adequate personal protective equipments. All patients with history of foreign body in aero digestive tract were advised preoperative $\mathrm{x}$-ray to know the presence, site, size \& type of foreign body.

Patients who had history of foreign body inhalation \& compromised airway in form of immediate endotracheal intubation were subjected to pre operative HRCT of neck and chest with virtual bronchoscopy to confirm the diagnosis. The CT scan helped us in reaching the diagnosis say presence of foreign body, size and site of its lodgement. Pre operative screening for covid-19 was done $24 \mathrm{~h}$ prior to procedure by RT-PCR test before airway intervention when patient's clinical condition was stable. Based on clinical and radiological diagnosis these patients were later taken up for direct laryngoscopy, bronchoscopy or tracheostomy as per the indications under general anesthesia in operation theatre. Full personal protective kit, which includes water impermeable gown, head cover, shoe cover, goggles, faceshield, N95mask and double gloves used by all staff members present in operation theatre during all procedures irrespective of covid-19 status of patient. All the procedures were performed by team comprising of a consultant E.N.T. surgeon, one assistant surgeon, two anaesthetists, one nursing staff and one operation theatre assistant. Precautions were taken to reduce aerosol generation during intubation, surgery and extubation. Patient was fully paralysed by anesthetist during tracheostomy, thorough suction was done from endotracheal tube and oropharynx before keeping incision on trachea. We also practised withhold positive pressure ventilation during making incision and insertion of the tracheostomy tube and minimal use of electrocautery.

Ear and nose foreign bodies which can be managed under local anesthesia were removed in minor procedure room with the help of endoscope or microscope. All data were collected \& analysed.

\section{Results}

The present study was carried out in Department of ENT and Head-Neck surgery, Medical College Baroda during covid-19 pandemic from 22/03/2020 to 30/06/2020. Total 29 cases were included.

Included patients were between 0 to 13 years of age. Youngest patient was of 1 day old neonate. Majority of patients were of 0 to 3 years of age. Among them 16 were male and 13 were female with male to female ratio of 1.23:1. (Table 1).

Out of 29 cases 10 had compromised airway with a presenting complaint of stridor either due to foreign body inhalation or due to underlying pathology. 5 patients had dysphagia as presenting complaint due to foreign body ingestion or oesophageal narrowing. (Fig. 1) (Table 2).

Tracheostomy was done in three patients for long standing intubation, videolaryngoscopy also done in these same three patients for diagnostic and therapeutic purpose. Cricothyrodotomy was done to secure airway due to failed intubation in one neonate. Another neonate had congenital anomaly (BOSMA Arhinia microophthalmia syndrome chromosome 18q11), nasopharyngeal airway secured through mouth in this patient. (Table 3) (Fig. 2) (Fig. 3).

Preoperative HRCT neck \& chest was done in 6 cases as screening and diagnostic tool. Among these 6 cases 3 had no obvious history of FB inhalation, 2 had history of extubation failure and 1 had failure in tracheostomy tube wean off. Among these 6 patients, bronchoscopy with foreign body removal from lower airway done in 3 patients and tracheostomy with videolaryngoscopy done in another 3 patients. None of the patient had classical covid-19 ground glass opacity on HRCT chest. Pre operative RT PCR covid-19 screening was done in 10 cases $\&$ all 10 cases were tested negative.

\section{Discussion}

This covid-19 pandemic time is challenging for all health care workers especially those who are involved in aerosol generating procedures. Before this pandemic no one was experienced to deal with such disease, so learning new methods and to adopt new circumstances was necessary. Developing new protocols will help not only in this pandemic but it will guide us \& new generation of health care workers in such pandemic conditions in future also. During pandemic at our institute we came across many pediatric emergencies. As an apex institute, we treated some of cases coming far away from other states with long standing pathologies and previously addressed by the specialist. 
Table 1 Clinical presentation of Patients

\begin{tabular}{ll}
\hline Clinical Presentation & No of patients \\
\hline Stridor & 10 \\
Dysphagia & 5 \\
FB ear & 5 \\
FB nose & 9 \\
\hline
\end{tabular}

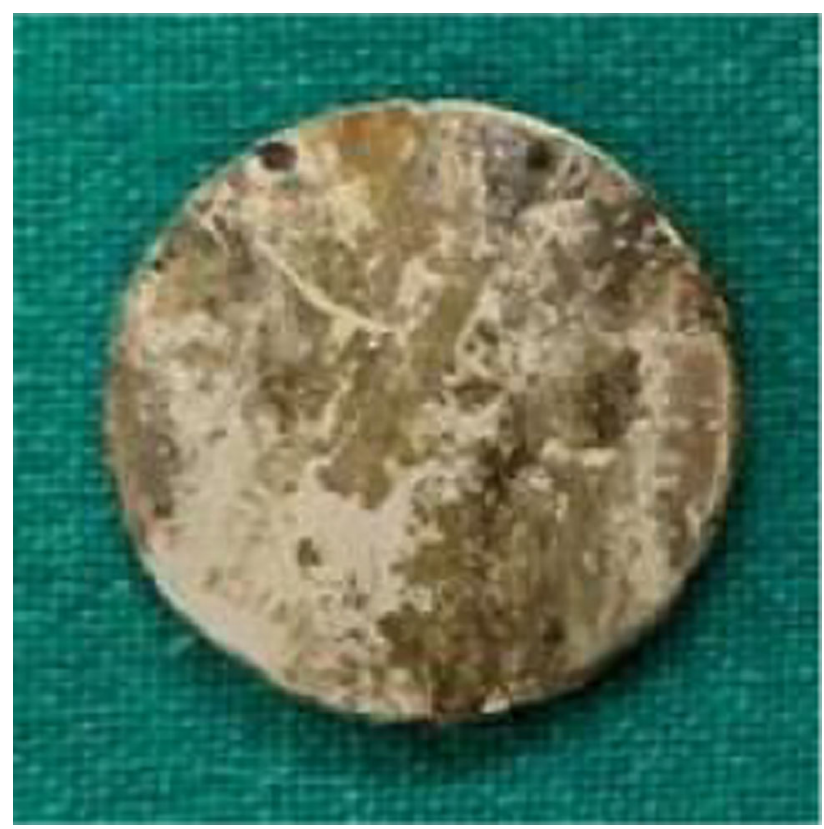

Fig. 1 Foreign body battery cell

Table 2 Types of procedure performed

\begin{tabular}{ll}
\hline Procedure & No of cases \\
\hline Bronchoscopy & 6 \\
Videolaryngoscopy & 3 \\
Tracheostomy & 3 \\
Cricothyrodotomy & 1 \\
Oesophagoscopy & 5 \\
Nasal FB removal & 9 \\
FB ear removal & 5 \\
\hline
\end{tabular}

SARS-CoV-2 is predominantly transmitted by contact or droplet transmission. It can become aerosolised by aerosol generating procedures and then airborne transmission is possible. We recommend use of adequate personal protective equipments in all the procedures irrespective of covid-19 testing in this pandemic era. Appropriate use of personal protective equipments significantly reduces the risk of viral transmission and infection [4]. Careful selection of patient is most important factor. Emergency cases, as well as cases where delay in intervention can lead to complications \& life threatening conditions must be addressed early. Similar recommendations suggested by Bann et al. in best practice recommendations for pediatric otolaryngology during the covid 19 pandemic [6].

In study done by Nicolas Leboulanger et.al, nonurgent surgeries should be postponed for at least 15 days (usual duration of quarantine in infected subjects) [7].

Pre operative screening of covid-19 by RT-PCR is desirable in current pandemic situation for safety of health care workers \& patients because majority of cases are asymptomatic. It is advisable to do testing within $24 \mathrm{~h}$ prior to the procedure if patient's clinical condition is stable. Pre operative covid-19 testing was not done whenever patient's clinical conditions and type of underlying pathology (foreign body battery cell) did not allow for any delay. Rapid antigen testing kit can be used as preoperative screening for covid-19 before such emergency procedures. In study done by Nicolas Leboulanger et al. Guidelines of the French Association of Pediatric Otorhinolaryngology (AFOP) and French Society of Otorhinolaryngology (SFORL) the nasopharyngeal swab with RT-PCR performed within $48 \mathrm{~h}$ preoperatively, unfortunately demonstrates 30 to $40 \%$ of false negative. It is, however, always useful. It becomes necessary if suggestive signs of Covid-19 infection are present. Urgent procedures should not be delayed by the expectancy of the results such as severe or progressive dyspnea, haemorrhages or severe infections [7].

In study conducted at children's hospital of Philadelphia gathered covid-19 screening data on preoperative 1295 pediatric patients, the overall incidence of covid-19 was $0.93 \%$. Among those pediatric patients who tested positive, half had no symptoms. Of those who did have symptoms, the most common were fever and a runny nose. The researchers noted symptoms were not useful in differentiating those who tested positive for covid-19 and those who tested negative [8]. Preoperative HRCT of neck \& chest in airway procedures is advisable if time \& condition of patient permits because it helps in diagnosis as well as in planning procedure and also reduces chances of negative scopy in suspected cases of foreign body inhalation. HRCT chest has great role in early detection of changes of pneumonia with ground glass appearance which are typical changes occurring in covid-19 patient. So it acts as screening tool in symptomatic patients. RT-PCR for diagnosis of SARS-CoV-2 infection alone has sensitivity of 71 to $80 \%$ [9]. Combined with HRCT chest its sensitivity increases to $90 \%$ [10]. Our findings are correlating with Nicolas Leboulanger et.al,Guidelines of the French Association of Pediatric Otorhinolaryngology (AFOP) and French Society of Otorhinolaryngology (SFORL) [7]. The 
Table 3 HRCT Neck and Thorax Findings

\begin{tabular}{ll}
\hline Findings & No of cases \\
\hline FB with lung pathology & 2 \\
Only FB & 1 \\
Only lung pathology & 1 \\
Airway narrowing & 2
\end{tabular}

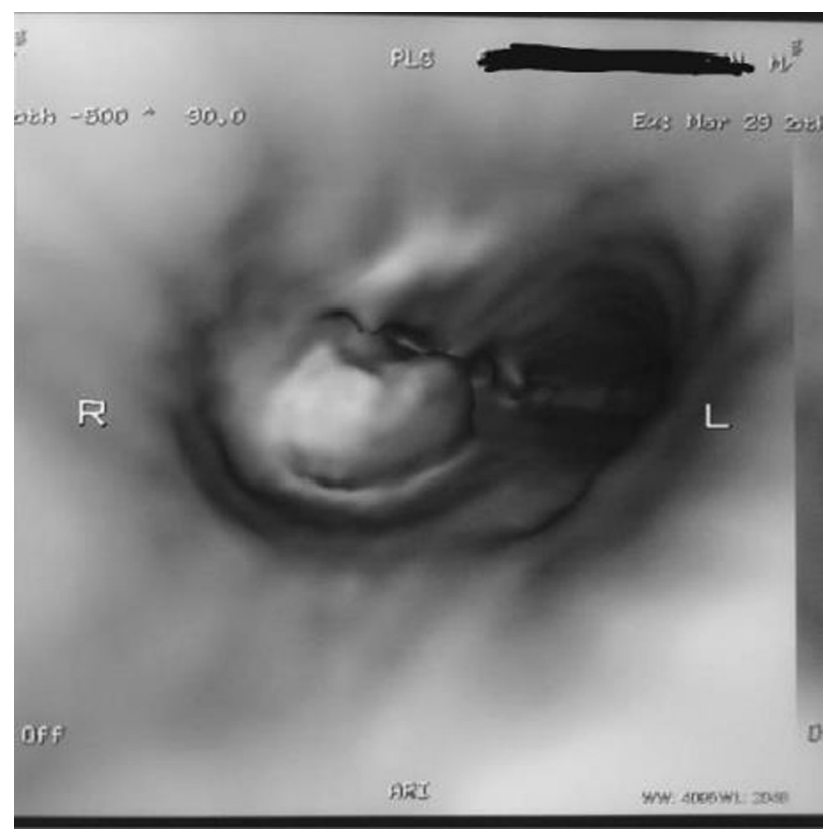

Fig. 2 Foreign body at right main bronchus

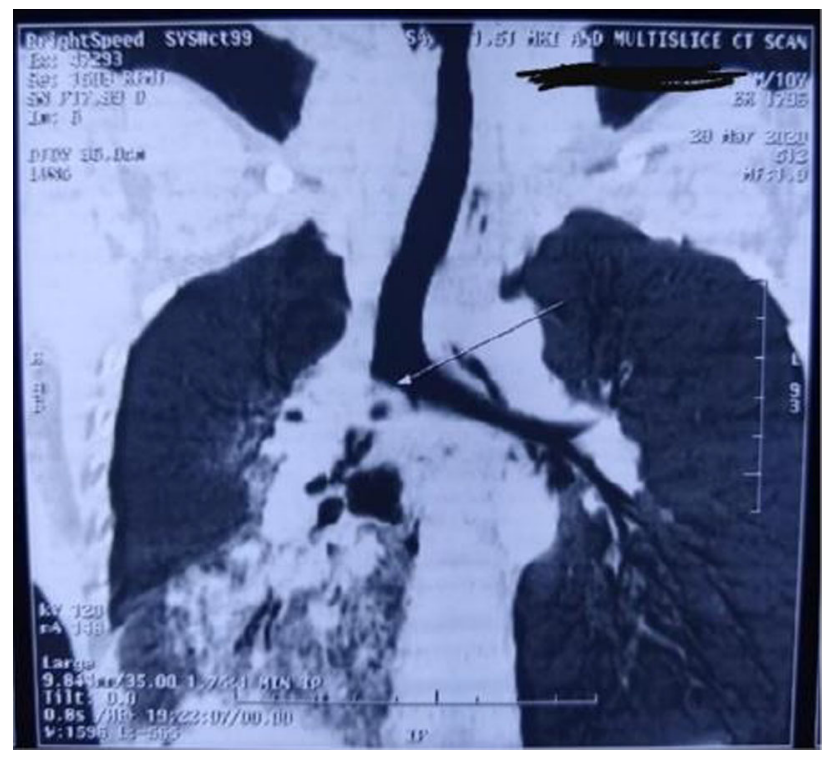

Fig.3 Foreign body at right main bronchus positive clinical diagnosis of bronchial foreign body in children is often difficult. When classical symptoms of foreign body aspiration are absent foreign body can often be missed. Such patients in absence of definite clinical diagnosis are treated as pneumonia, laryngitis and COPD causing delayed in diagnosis and eventually land up with complications [11]. Chest radiography is usually the first diagnostic study ordered, with a reported sensitivity and specificity of $72 \%$ to detect abnormalities consistent with a foreign body aspiration. Recent progress in medical imaging, with the development of MDCT has decreased the acquisition time and improved image quality. 3D images on the basis of MDCT can provide virtual tracheobronchoscopy and confirmation of bronchial foreign body. In absence of such imaged radiological findings any interventional procedures can be avoided in current pandemic era. MDCT can be performed in children with minimal radiation risks:[12].

We recommend necessary preparations in Operation Theater related to anesthesia \& surgery before shifting patient. During preparation, intra operatively as well post operatively the principle is to decrease aerosolization and droplet contamination in the operating room and recovery room. Similar guidelines recommended by Claire Frauenfelder et al. to reduce number of health care workers exposure [13]. David J Brewster et al. recommended negative pressure ventilation rooms are ideal to minimise exposure to aerosol and droplet particles. Where this is not feasible, normal pressure rooms with closed doors are recommended [14].

Precautions taken to reduce aerosol generation inside operation theatre during preparation and surgery were to sedate patients before induction, snugly fitting face mask used for oxygenation, immediately cuff inflation after endotracheal tube insertion and attachment of filter with close circuit systems is used. Similar recommendation was also suggested by Shama A Bellad et al. during intubation and extubation to prevent risk of aerosalisation of viral particle [15]. The lowest gas flow to maintain ventilation with face mask was used during open airway procedures to minimize aerosol spread. We used transparent drapes in videolarynoscopies and during tracheostomies and used optical bronchoscope for removal of foreign body from lower airway. Patient was fully paralysed by anesthetist during tracheostomy, thorough suction was done from endotracheal tube and oropharynx before keeping incision on trachea. We also practised withhold positive pressure ventilation during making incision and insertion of the tracheostomy tube and minimal use of electrocautery. Periodically cleaning of Operation Theater surface \& equipments by using $1 \%$ sodium hypocholride and also fogging done using hydrogen peroxide diluted in silver nitrate solution in Operation Theater. This will reduce infection transmission due to fomites. These procedures 
were done at normal room pressure with closed doors, negative OT room pressure is preferred if available.

Study by Michael J Brenner et al. also suggested complete paralysis to eliminate patient movement and coughing, maintenance of a bloodless field, minimal use of diathermy, use of a smoke evacuator, pausing of positive pressure ventilation during insertion of the tracheostomy tube [16]. Indications of tracheostomy should be restricted to cases with no other therapeutic solution. Sample size and wide variety of patients are main Strength of this study changing in treatment protocol for COVID over period of time are few limitations.

\section{Conclusions}

Preoperative covid-19 screening by RT-PCR test is advisable to detect active case prior to aerosol generating airway procedures and in life threatening situations a rapid antigen testing kit is recommended. HRCT neck and chest with virtual bronchoscopy is helpful not only for diagnosis of underlying pathology but also act as screening tool for covid-19.

Comprehensively we recommend only emergency procedures to be performed during pandemic, use of personal protective equipment during all procedures, preoperative investigations for diagnosis and modifications in operation theatre, anesthesia \& surgical technique procedure to reduce aerosols will minimise risk of infection transmission to health care workers.

\section{Compliance with Ethical standards}

Conflict of interest All the authors declare that they have no conflict of interest in the submission of this Manuscript.

Human and Animal Rights This article does not contain any studies with animals performed by any of the Authors.

Ethical Approval All procedures performed in the studies involving human participant were in accordance with the ethical standards of the institutional research committee and ethical standards.

Informed Consent Informed consent was obtained from all individual participants included in the study.

\section{References}

1. Huang C, Wang Y, Li X et al (2020) Clinical features of patients infected with 2019 novel coronavirus in Wuhan. China Lancet 395:497-506. https://doi.org/10.1016/S0140-6736(20)30183-5

2. WHO: Modes of transmission of virus causing COVID-19: implications for IPC precaution recommendations. https://www. who.int/news-room/commentaries/detail/modes-of-transmissionof-virus-causing-covid-19-implications-for-ipc-precautionrecommendations (accessed 15th May 2020).
3. Van der Poel N, Mansbach AL, Loundon N, Russell J, Vanderveken OM, Boudewyns A (2020) Challenges in pediatric otolaryngology in the COVID-19 pandemic: insights from current protocols and management strategies. B-ENT 16(1):59-62

4. Cook TM (2020) Personal protective equipment during the coronavirus disease (COVID) 2019 pandemic-a narrative review. Anesthesia 75:920-927. https://doi.org/10.1111/anae.150715

5. Hariga I, Khamassi K, Zribi S, Amor MB, Gamra OB, Mbarek C, Khedim AE (2014) Management of foreign bodies in the Aero digestive tract. Indian $\mathrm{J}$ Otolaryngol Head Neck Surg 66(1):220-224. https://doi.org/10.1007/s12070-011-0436-y

6. Bann DV, Patel VA, Saadi R, Goyal N, Gniady JP, McGinn JD, Goldenberg D, Isildak H, May J, Wilson MN (2020) Best Practice Recommendations for Pediatric Otolaryngology during the COVID-19 Pandemic. Otolaryngol Head Neck Surg 162(6):783-794. https://doi.org/10.1177/0194599820921393

7. Leboulanger N, Sagardoy T, Akkari M, Ayari-Khalfallah S et al. (2020) COVID-19 and ENT Pediatric otolaryngology during the COVID-19 Pandemic. Guidelines of the French Association of Pediatric Otorhinolaryngology (AFOP) and French Society of Otorhinolaryngology (SFORL). Eur Ann Otorhinolaryngol Head Neck Dis 137(3): 177-181 https://doi.org/10.1016/ j.anorl.2020.04.010

8. Parikh SR, Bly RA, Bonilla-Velez J et al (2020) Pediatric Otolaryngology Divisional and Institutional Preparatory Response at Seattle Children's Hospital after COVID-19 Regional Exposure. Otolaryngol Head Neck Surg 162(6):800-803. https://doi.org/ 10.1177/0194599820919748

9. Hani C, Trieu NH, Saab I, Dangeard S, Bennani S, Chassagnon G, Revel M-P (2020) COVID-19 pneumonia: A review of typical CT findings and differential diagnosis. Diagn Interv Imaging 101(5):263-268. https://doi.org/10.1016/j.diii.2020.03.014

10. Ai T, Yang Z, Hou H et al (2020) Correlation of Chest CT and RT-PCR Testing for Coronavirus Disease 2019 (COVID-19) in China: A Report of 1014 Cases. Radiology 296(2):E32-E40. https://doi.org/10.1148/radiol.2020200642

11. Hassan MA, Youssef TA, El-Gharib M (2013) Role of virtual bronchoscopy in foreign body inhalation in children. Egypt $\mathrm{J}$ Otolaryngol 29(4):249-253. https://doi.org/10.7123/01.EJO. 0000433256.86712 .83

12. Paterson A, Frush DP (2007) Dose reduction in PEDIATRIC MDCT: general principles. Clin Radiol 62:507-517. https://doi.org/10.1016/j.crad.2006.12.004

13. Frauenfelder C, Butler C, Hartley B et al (2020) Practical insights for PEDIATRIC otolaryngology surgical cases and performing microlaryngobronchoscopy during the COVID-19 pandemic. Int J Pediatr Otorhinolaryngol 134:110030. https://doi.org/10. 1016/j.ijporl.2020.110030.10.1016/j.ijporl.2020.110030

14. Brewster DJ, Chrimes N, Do TB et al (2020) Consensus statement: Safe Airway Society principles of airway management and tracheal intubation specific to the COVID-19 adult patient group. Med J Aust 212(10):472-481. https://doi.org/10.5694/mja2.50598

15. Shama A Bellad, Priti S Hajare, Rajesh Radhakrishna Havaldar et al. Challenges in the management of ENT foreign bodies during COVID-19 Pandemic\&nbsp;-Experience at a tertiary care centre., 24 August 2020, PREPRINT (Version 1) available at Research Square https://doi.org/10.21203/rs.3.rs-57151/v1+]

16. Michael J Brenner, Stephen J Warrillow, Vinciya Pandian, Asit Arora, Tanis S Cameron et al (2020) Tracheostomy in the COVID-19 era: global and multidisciplinary guidance 8(7): 717-725 https://doi.org/10.1016/S2213-2600(20)30230-7

Publisher's Note Springer Nature remains neutral with regard to jurisdictional claims in published maps and institutional affiliations. 